\title{
Emotion-attention network interactions during a visual oddball task
}

\author{
Harlan M. Fichtenholtz ${ }^{\mathrm{a}}$, Heather L. Dean ${ }^{\mathrm{b}}$, Daniel G. Dillon ${ }^{\mathrm{a}}$, Hiroshi Yamasaki ${ }^{\mathrm{c}}$, \\ Gregory McCarthy ${ }^{\mathrm{d}}$, Kevin S. LaBar ${ }^{\mathrm{a}, *}$ \\ ${ }^{a}$ Center for Cognitive Neuroscience, Duke University, Room B203, LSRC Building, Durham, NC 27708-0999, USA \\ ${ }^{\mathrm{b}}$ Department of Neurobiology, Duke University Medical Center, Durham, NC 27710, USA \\ ${ }^{\mathrm{c}}$ Department of Integrative Physiology, National Institute for Physiological Sciences, Okazaki 444-0806, Japan \\ ${ }^{\mathrm{d}}$ Brain Imaging and Analysis Center, Duke University Medical Center, Durham, NC 27710, USA
}

Accepted 28 January 2004

Available online 16 March 2004

\begin{abstract}
Emotional and attentional functions are known to be distributed along ventral and dorsal networks in the brain, respectively. However, the interactions between these systems remain to be specified. The present study used event-related functional magnetic resonance imaging (fMRI) to investigate how attentional focus can modulate the neural activity elicited by scenes that vary in emotional content. In a visual oddball task, aversive and neutral scenes were presented intermittently among circles and squares. The squares were frequent standard events, whereas the other novel stimulus categories occurred rarely. One experimental group $[N=10]$ was instructed to count the circles, whereas another group $[N=12]$ counted the emotional scenes. A main effect of emotion was found in the amygdala (AMG) and ventral frontotemporal cortices. In these regions, activation was significantly greater for emotional than neutral stimuli but was invariant to attentional focus. A main effect of attentional focus was found in dorsal frontoparietal cortices, whose activity signaled task-relevant target events irrespective of emotional content. The only brain region that was sensitive to both emotion and attentional focus was the anterior cingulate gyrus (ACG). When circles were task-relevant, the ACG responded equally to circle targets and distracting emotional scenes. The ACG response to emotional scenes increased when they were task-relevant, and the response to circles concomitantly decreased. These findings support and extend prominent network theories of emotion-attention interactions that highlight the integrative role played by the anterior cingulate.

(C) 2004 Elsevier B.V. All rights reserved.
\end{abstract}

Theme: Neural basis of behavior

Topic: Motivation and emotion

Keywords: Emotion; Attention; Anterior cingulate gyrus; Functional MRI; Target detection; Novelty

\section{Introduction}

Emotional processing and attentional control have been attributed to two large-scale neural networks. Stimuli with emotional content engage fronto-limbic structures, including the amygdala (AMG) and ventral prefrontal cortex, and modulate activity along hierarchically organized sensory processing streams (for review, see Refs. $[1,46]$ ). In contrast, a network of regions in posterior parietal and dorsolateral prefrontal cortices mediate attentional control (for review, see Ref. [14]). A common link between these two distrib-

\footnotetext{
* Corresponding author. Tel.: +1-919-681-0664; fax: +1-919-681-0815.

E-mail address: klabar@duke.edu (K.S. LaBar).
}

uted systems is the cingulate cortex, which may play a unique role in emotion-attention interactions.

The representation and integration of emotional and attentional information within the cingulate cortex is not well understood. Nimchinsky et al. [42] have described a specific neuronal phenotype (spindle neurons) in the anterior cingulate gyrus (ACG) and distributed along its length that is hypothesized to link emotional and cognitive functions. Others have defined functional subdivisions within the cingulate cortex that are specialized for different aspects of behavior [6,12,55,59]. For example, Mega et al. [36] suggest that the cingulate cortex consists of four functional components - a visceral effector, a cognitive effector, a skeletomotor effector, and a sensory processing region. Allman et al. [2] posit that although affective and cognitive tasks produce 
different foci of cingulate activity, the spatial extent of the resulting activations overlap and the regions may not be completely distinct. Furthermore, spindle neurons are found in both the affective and cognitive divisions of the ACG as defined functionally [7].

Mayberg [34] has suggested that ventral cortical and subcortical regions which constitute much of the emotional processing network are specialized for "vegetative-somatic" functions, whereas dorsal areas included in the attentional orienting network are specialized for "attentional-cognitive" functions. These networks follow and extend the dorsal and ventral streams of visual processing (e.g., Ref. [20]). Mayberg hypothesizes that the rostral portion of the ACG serves as an intermediary between these processing streams. Mesulam [37] also postulates that the cingulate cortex is critical for the integration of emotional or motivational relevance with attention due to its connections between limbic structures and sensory association cortices. Finally, the ACG was central to Papez's [43] theory of emotion, where the cingulate was viewed as the "seat of dynamic vigilance," the location of integration between emotional and executive processing.

Although clinical observations and animal models of cingulate neglect have supported some aspects of these anatomical theories $[21,37,39,43,58]$, few functional imaging studies have directly investigated the relationship between emotional and attentional processing systems in the brain. The existing studies have generally taken two approaches to this interaction. The first approach has investigated how attentional resources are allocated when there is competition between task-relevant stimulus attributes and concurrent emotional information that elicits a prepotent sensory or response bias. For instance, Stroop interference tasks appear to engage different subdivisions of ACG depending on whether the target stimuli are aversive or neutral, with emotional Stroop effects localized to more ventral regions of the ACG $[6,59]$. Other studies have investigated the processing of emotional facial expression under conditions of attention and inattention during spatial cueing tasks in which other neutral stimuli were simultaneously presented. Vuilleumier et al. [56] showed that amygdala responses to displays of facial affect were not modulated by visuospatial attention, but responses in other brain regions (e.g., fusiform gyrus) were. In contrast, Pessoa et al. [45] showed that responses to emotional faces in both the amygdala and fusiform gyrus were modulated by attention when resources were presumably more exhausted. Both of these studies showed an interaction between emotional and attentional processing in the ACG.

The second approach has been to investigate how the neural representation of an emotional stimulus changes when participants selectively attend to their emotional responses to the stimulus versus some other stimulus features (e.g., spatial attributes). Lane et al. [28] showed that when participants attended to their own emotional responses (pleasant/neutral/ unpleasant) to a stimulus, there was greater ACG activity than when participants were attending to the spatial setting of the stimulus (indoor/outdoor/either). In an additional study, Lane et al. [30] reported activity in the ACG that co-varied positively with the participants' emotional awareness (see also Ref. [31]). This could be interpreted as participants exhibiting greater ACG activity when they attended to the emotional aspect of a stimulus to a greater extent.

The visual oddball task is another experimental paradigm that is well suited to investigate the relationship between emotional and attentional processing. In a typical oddball paradigm, participants detect rare task-relevant target events embedded in a sequential stream of frequent standard stimuli [11]. In this task, the direction of one's attentional focus over time is assigned to a specific, rarely occurring stimulus category that is deemed task-relevant. Whenever an exemplar of this stimulus category appears, participants are asked to initiate a motor response and/or mentally count the number of oddball exemplars. The introduction of equally rare, task-irrelevant stimuli (distracters) to oddball tasks allows for the manipulation of attentional focus across different stimulus categories.

Thus, by presenting infrequent, emotionally evocative stimuli in an oddball task and varying whether those stimuli serve as attentional targets or distracters, one can investigate the effect of attentional focus (as defined by task relevancy) on emotional stimulus processing. In a previous eventrelated functional magnetic resonance imaging (fMRI) study [60], we asked participants to discriminate infrequent novel targets (circles) embedded within a stream of frequent standard stimuli (squares) and two categories of novel, task-irrelevant distracters (aversive and neutral scenes). Activation was seen in the amygdala and associated ventral stream regions in response to the task-irrelevant aversive scenes but not the attentional targets. The opposite pattern was found in the dorsal frontoparietal cortex. The one region that responded to both attentional targets and emotional distracters was the ACG.

The present study was designed as a follow-up to the Yamasaki et al. [60] study in which we reversed the emotional salience of the targets and novel distracters. In our previous study, attentional focus and emotional information processing were segregated in time across different categories of stimuli. Here, we contrast this condition, where circles were task-relevant (Group 1) with another group of participants for whom the emotional scenes were task-relevant (Group 2). Consequently, for participants in Group 2, attentional focus and emotional processing converged on a common target stimulus. Comparing results across the two groups will enable a more comprehensive understanding of the neural substrates that mediate the interaction between emotional salience and attentional focus.

The present experiment tested several hypotheses. First, due to the convergence of attentional focus and emotional processing on a single stimulus, the aversive scenes should recruit both ventral emotional and dorsal attentional networks for participants in Group 2. Second, some research suggests 
that the ability of emotional stimuli to elicit activity in ventral stream regions is relatively automatic [31] (see Ref. [46] for review) and should not be affected by task relevancy (but see Ref. [45]). Consequently, the response of ventral stream regions to emotional stimuli should be equivalent across Groups 1 and 2. Third, the results of Yamasaki et al. [60] suggest that the processing of emotional distracters and attentional targets within the oddball paradigm occurs in the same broad region of the ACG. Therefore, when the emotional scenes are task relevant (i.e., in Group 2), attentional and emotional processing should be integrated and engender more ACG activity than in Group 1, where these functions are distributed across different stimulus categories over time. Finally, we test whether the oddball response in dorsal brain regions is invariant to emotional content. Because dorsal frontoparietal cortex is consistently activated in oddball tasks that have used a variety of target stimuli $[8,9,25,35,49]$, we expect to see similar activity in these regions across the two experimental groups.

\section{Method}

\subsection{Participants}

Twenty-nine healthy right-handed adults were randomly assigned to one of two groups. Group 1 consisted of 13 participants. Data from three participants were not analyzed due to excessive head movement. Head motion was analyzed using a center of mass measurement, and participants were discarded if they showed more than $3 \mathrm{~mm}$ of movement in the $X, Y$, or $Z$ planes. The analyzed group consisted of 10 participants (four males). Some of the region-of-interest (ROI) data from this group were previously reported by
Yamasaki et al. [60]. Group 2 consisted of 16 participants. Data from four participants were not analyzed due to excessive head movement. The analyzed group consisted of 12 participants (nine males). The participants ranged in age from 20 to 47 years old and were compensated for their participation at a rate of US\$20.00 per hour. Participants were screened for a history of neurological and psychiatric disorders, substance abuse, current medications, and safety for MRI procedures. The Duke University Medical Center Institutional Review Board approved the protocol for this study.

\subsection{Task parameters}

The MRI session consisted of 10 task runs. Each run contained 132 stimuli presented centrally. Stimuli were presented every $3000 \mathrm{~ms}$ for a duration of $2000 \mathrm{~ms}$. During the 1000-ms period between stimuli, a fixation cross was presented. There were four stimulus categories: squares of various sizes and colors, circles of various sizes and colors, aversive scenes, and neutral scenes. Squares were the standard stimulus, occurring on $84.4 \%$ of trials. Circles occurred on $8 \%$ of trials, and aversive and neutral scenes occurred on $3.8 \%$ of trials each. No individual circle or scene was repeated during the session. Circles, aversive and neutral scenes were pseudorandomly distributed throughout each task run and separated by $12-21 \mathrm{~s}($ mean $=18 \mathrm{~s})$. Within each session, a participant viewed a total of 106 circles, 50 aversive scenes and 50 neutral scenes. The task design is illustrated in Fig. 1.

The participant's task differed by group. Participants in Group 1 were instructed to press a button with their right index finger if they detected a circle on the screen (taskrelevant target) and to press another button with their right middle finger for all other stimuli. Thus, motor responses were required of all stimuli to avoid potential motor prepa-

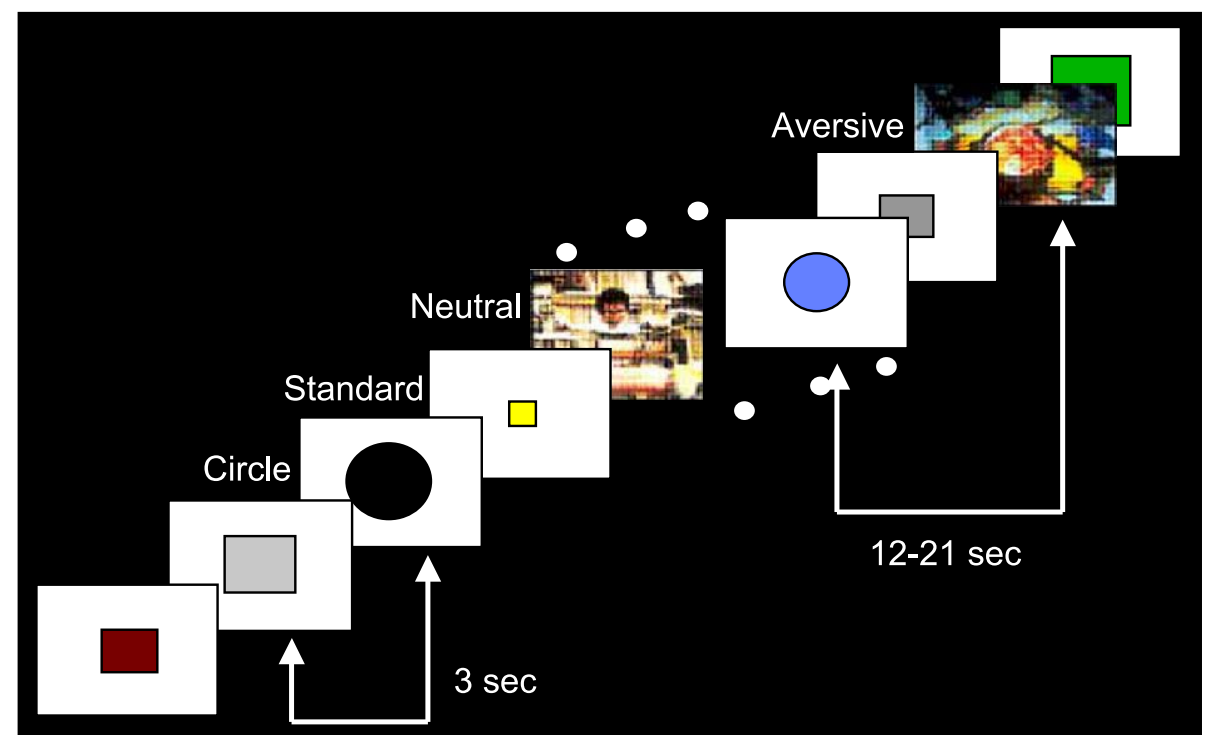

Fig. 1. This variation on the visual oddball task used squares as the standard stimulus and circles, aversive and neutral scenes as infrequent stimuli. A stimulus was presented every $3 \mathrm{~s}$, and there was a minimum of $12 \mathrm{~s}$ between any two infrequent stimulus exemplars. 
ration and execution confounds across stimulus categories. Participants were also instructed to keep a mental count of the number of targets, which was reported to the experimenter at the end of each run. The instructions for participants in Group 2 were identical, except that they pressed a button and kept a mental count of aversive scenes (task-relevant target). To re-emphasize, the only difference between the two experimental groups was the assignment of the task-relevant target category. All sensory stimuli, response requirements and task parameters were identical across the groups.

\subsection{Stimuli}

Aversive scenes were selected from the International Affective Picture System (IAPS) [33] and depicted human violence, mutilation, and disease. Neutral scenes depicted everyday activities and were compiled from the IAPS set and in-house sources to equate the stimulus categories for mean luminance and chromatic features. Mean luminance was adjusted to fall between 112.5 and 113.5 luminance units with a S.D. between 62.5 and 63.5 using Photoshop software (Adobe Systems, San Jose, CA). All of the scenes contained human figures or body parts. The scenes were selected according to ratings for arousal $(1=l o w, 9=h i g h)$ and valence ( 1 =negative, 9 =positive). Ratings were provided in the IAPS norms and from a pilot group of participants. Arousal ratings were between 5 and 8 for aversive scenes and between 1 and 3 for neutral scenes. Valence ratings were between 1 and 3 for aversive scenes and between 4 and 6 for neutral scenes. By using these ranges, there was no overlap between the ratings for the two groups of scenes. The circles and squares were trial-unique and varied in their size and color.

\subsection{MRI acquisition}

Images were acquired using a 1.5T General Electric NVi scanner (Milwaukee, WI) equipped with $41 \mathrm{mT} / \mathrm{m}$ gradients. Head movement was minimized by supporting the participant's head in a vacuum cushion. A localizer series was acquired to identify the anterior (AC) and posterior (PC) commissures. A high-resolution T1-weighted structural series was acquired parallel to the $\mathrm{AC}-\mathrm{PC}$ plane (34 slices, $\mathrm{TR}=450 \mathrm{~ms}, \mathrm{TE}=20 \mathrm{~ms}, \mathrm{FOV}=24 \mathrm{~cm}$, matrix $=256^{2}$, slice thickness $=3.75 \mathrm{~mm}$ ). A second T1weighted series was acquired perpendicular to the $\mathrm{AC}-\mathrm{PC}$ plane using the same parameters. Gradient echo echoplanar images ( 34 slices, $\mathrm{TR}=3000 \mathrm{~ms}, \mathrm{TE}=40 \mathrm{~ms}, \mathrm{FOV}=24 \mathrm{~cm}$, matrix $=64^{2}$, flip angle $=90^{\circ}$, slice thickness $=3.75 \mathrm{~mm}$; resulting in $3.75 \mathrm{~mm}^{3}$ isotropic voxels) sensitive to bloodoxygenation-level-dependent contrast were acquired using the same prescription as the T1-weighted structural images.

\section{5. fMRI data analysis}

Two data analysis methods were used based on voxel-wise statistical parametric mapping (SPM) and anatomical ROI drawings. Anatomical ROIs were drawn for each participant individually based on high-resolution coronal anatomical images. ROIs were drawn using an in-house mouse driven computer program (Brain Imaging and Analysis Center, Duke University). The program ran within the Matlab environment (Mathworks, Natick, MA) on a PC-DOS platform. Anatomical constraints were defined for each participant individually, guided by the anatomical borders described in Talairach and Tournoux [52] and Duvernoy [15]. Slices were indexed by their distance from the anterior commissure to allow the activations in these regions to be compared across participants. ROIs were drawn separately for each hemisphere. There were 14 ROIs drawn for each participant. The ROIs were drawn on a slice-by-slice basis for the middle frontal gyrus (MFG) on 8 slices, inferior frontal gyrus (IFG) on 8 slices, cingulate gyrus (CG) on 16 slices, intraparietal sulcus (IPS) on 12 slices, supramarginal gyrus (SMG) on 5 slices, FFG on 14 slices, and AMG on 3 slices. These procedures have been described previously by Jha and McCarthy [24] and Yamasaki et al. [60].

Volumes were corrected for their interleaved acquisition sequence using a cubic spline interpolation to realign each voxel's time course to the time-to-repetition (TR) onset. No spatial preprocessing was performed. Following the method of McCarthy et al. [35], epochs of interest were defined around the onsets of the circles, aversive and neutral scenes. Each epoch consisted of the two TRs prior to the stimulus (beginning at $-6 \mathrm{~s}$ ) and the five TRs after the stimulus (ending at $15 \mathrm{~s}$ ). The raw MR signal was extracted for each epoch and averaged for each stimulus type. An average of the MR signal for all of the voxels within each ROI was plotted to represent the experimentally derived hemodynamic response function for each ROI during each stimulus condition. The average MR signal values were converted to percent signal change relative to the average of the pre-stimulus baseline points. The only stimuli presented during the baseline period of each epoch were the standard stimuli, to allow responses from the novel stimulus categories to return to baseline. The interval between successive events of interest (targets, distracters) was sufficiently long ( $>12 \mathrm{~s}$ ) to minimize possible refractory effects $[22,23]$. In addition to the length of time between stimuli of interest, any remaining overlap was consistent across all stimulus types since event ordering was randomized. The rapid nature of the stimulus presentation provides a baseline for analyses comparing the novel stimulus categories to each other, which was our main interest a priori, but precludes identification of brain regions activated by the standards themselves. The percent signal change at the peak time point ( $6 \mathrm{~s}$ post-stimulus) was analyzed by repeatedmeasures analysis of variance (ANOVA) followed by post hoc comparisons using the Student-Neuman-Keuls test to further investigate the main effects due to stimulus category and group. An alpha level of 0.05 was used to determine significant differences in all contrasts.

Data were also analyzed using SPM99 [16] to identify regions of activation outside those hypothesized a priori. The 
functional images were corrected for their interleaved acquisition order and realigned to the first image to correct for head motion. The realigned images were then co-registered to the co-planar anatomical image for each participant. The coplanar anatomical images were spatially normalized to a common stereotactic space using the Montreal Neurological Institute (MNI) template included in SPM99. The functional images were then normalized to the common space using the parameters defined by the co-planar anatomical image and smoothed using an 8-mm isotropic Gaussian kernel.

The responses to the infrequent stimulus categories were isolated by convolving a vector of onset times of the circles, aversive and neutral scenes with a synthetic hemodynamic response function that emphasized transient activity in response to the events. The general linear model was used to model the effects of interest and other confounding effects, such as session effects or motion related artifacts, for each participant. Across participant comparisons were made using a random effects model which accounts for between participant variability and allows for the generalization of the results beyond the current group of participants. This is especially critical for between-subjects designs. Two conjunction analyses were performed by computing a composite image of the active voxels across the two experimental groups for aversive scenes and attentional targets. Conjunction images were calculated by finding voxels that reached a significant threshold of $p<0.001$ within each condition included in the conjunction, for an overall level of $p<0.000001$ across two conditions. Statistical maps were thresholded at the level of $p<0.001$ uncorrected for all analyses. The conjunction images provide information about the extent of the common activations, but not their intensity. To get this information, images were computed by multiplying the activation $T$-maps for each condition of interest with the original conjunction map. This procedure provides a combined $T$-value for each cluster of activation across both conditions. Coordinates of activated clusters were converted from the MNI template to the stereotactic space described by Talairach and Tournoux [52] according to methods described by Lancaster et al. [27].

\section{Results}

\subsection{Behavioral performance}

Due to technical problems, button response data from five subjects in Group 2 were not available for analysis; thus, analyses of behavioral performance were conducted on 10 participants from Group 1 and 7 participants from Group 2. Mean ( \pm S.E.M.) accuracy for target detection was high for both groups (Group 1: $93.5 \% \pm 1.3$; Group 2: $94.2 \% \pm$ 1.3). There was no significant difference in accuracy between the two groups $(t(15)=-0.34, p=0.74)$.

A two-way mixed ANOVA of reaction time (RT) data, including group and stimulus type as factors, revealed significant main effects of group and stimulus type and a significant group $\times$ stimulus type interaction $(F(1,15)=$ $5.49, p<0.03 ; F(3,45)=112.78, p<0.001 ; F(3,45)=51.78$, $p<0.001$, respectively). Post hoc $t$ tests across groups revealed that Group 2 showed significantly longer RTs in response to both the neutral and aversive scenes than Group $1(t(15)=4.99, p<0.001 ; t(15)=4.24, p<0.001$, respectively). Within Group 1, subjects took longer to respond to aversive than neutral scenes $(t(9)=2.95, p<0.02)$, with an intermediate RT to circles that was not significantly different from either category. Furthermore, all stimulus types showed longer RTs than standards. Within Group 2, RTs in response to aversive and neutral scenes did not differ and were longer than the RTs in response to circles and standards, which did not differ from each other. Means and S.E.M.s can be found in Table 1 .

These results suggest that participants in Group 2 were taking longer to perform the task in order to achieve the same level of accuracy. For both groups, RTs to the aversive and neutral scenes were longer than the RTs to the standard stimuli. This difference was also seen for the task-relevant circles for Group 1.

\section{2. fMRI results: ventral regions of interest}

\subsubsection{Amygdala and fusiform gyrus}

Within the AMG and FFG, there were main effects of stimulus type $(F(2,40)=15.05, p<0.001$ and $F(2,40)=$ $159.06, p<0.001$, respectively). Post hoc comparisons showed that aversive scenes generated larger responses than neutral scenes, which, in turn, elicited larger responses than circles (see Fig. 2).

\subsubsection{Inferior frontal gyrus}

A two-way ANOVA revealed a significant group $\times$ stimulus type interaction $(F(2,40)=4.26, p=0.021)$. A follow-up one-way ANOVA for Group 1, where circles were taskrelevant, revealed a significant main effect of stimulus type $(F(2,18)=22.37, p<0.001)$. Post hoc comparisons showed that aversive scenes generated a larger response than neutral

Table 1

Response times as a function of stimulus category

\begin{tabular}{lrc}
\hline & Mean & S.E.M. \\
\hline Group 1 & 517.3 & \\
Squares & 674.1 & 51.0 \\
Circles & 705.6 & 46.4 \\
Aversive scenes & 669.3 & 48.3 \\
Neutral scenes & & 45.7 \\
Group 2 & & \\
Squares & 575.1 & 42.7 \\
Circles & 571.9 & 43.6 \\
Aversive scenes & 1005.9 & 48.6 \\
Neutral scenes & 1020.9 & 53.1 \\
\hline
\end{tabular}

Circles were task-relevant for Group 1, and aversive scenes were taskrelevant for Group 2. 
Ventral Regions

Group 1

Circle Targets
Group 2

Aversive Targets

A

AMG

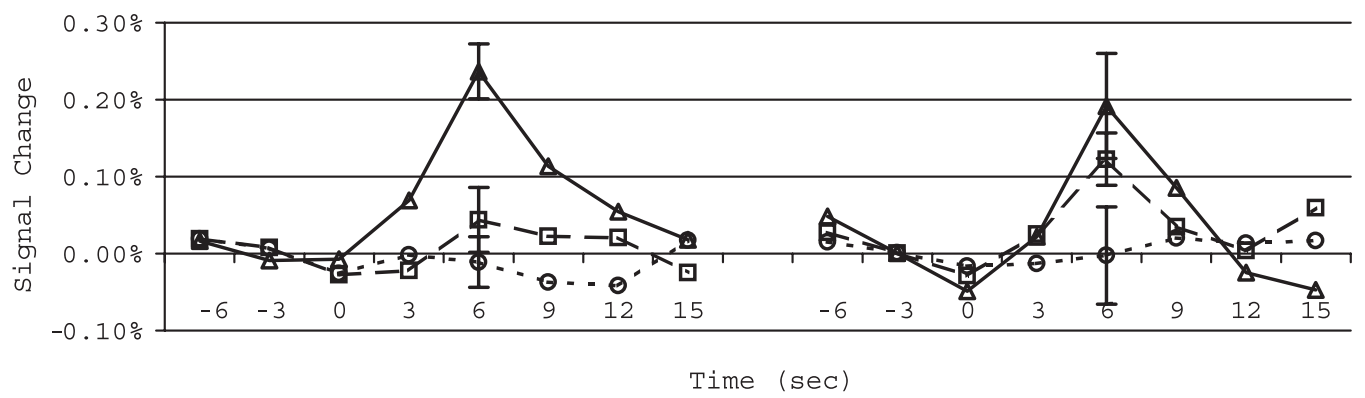

B

FFG

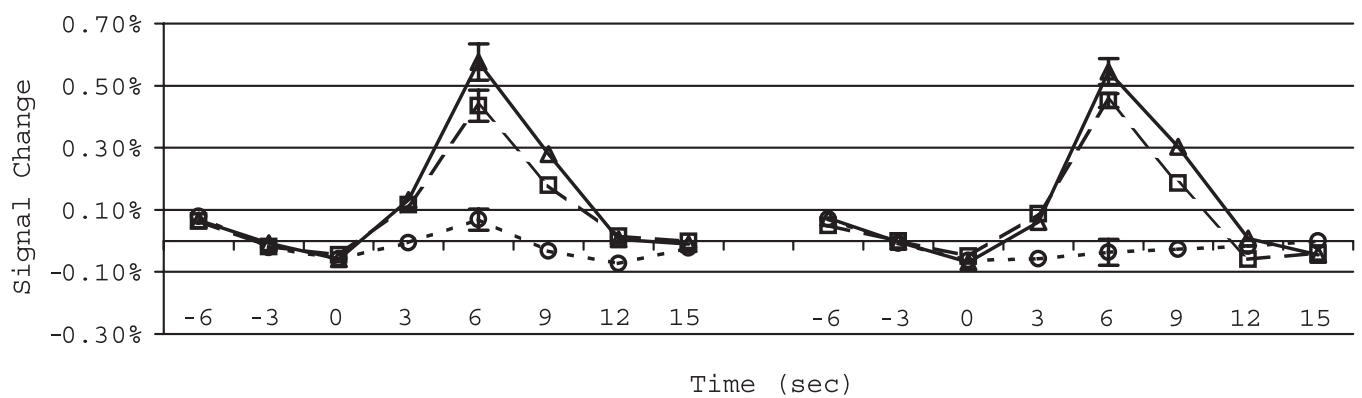

C

IFG

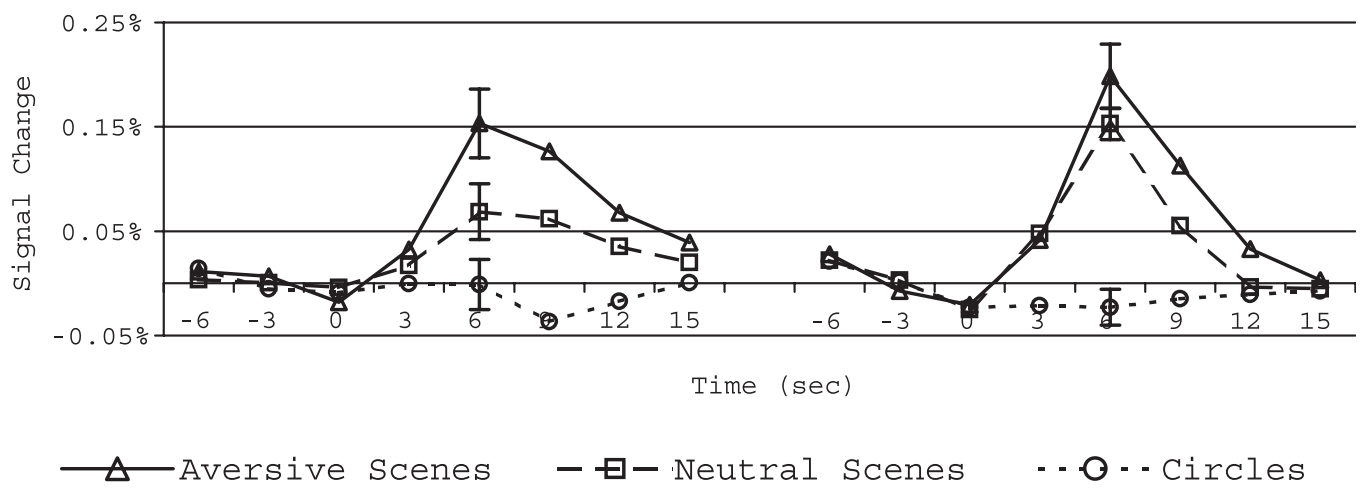

Fig. 2. Activation in the ventral regions of interest by group averaged across left and right hemispheres. Circles were task-relevant for Group 1, whereas aversive scenes were task-relevant for Group 2. Response within the amygdala (A), fusiform gyrus (B), and inferior frontal gyrus (C) to circles, aversive scenes, and neutral scenes is depicted.

scenes, which, in turn, generated a larger response than circles. A follow-up one-way ANOVA for Group 2, where aversive scenes were task-relevant, revealed a significant main effect of stimulus type $(F(2,22)=36.31, p<0.001)$. Post hoc comparisons showed that both aversive and neutral scenes generated a larger response than circles (see Fig. 2).

\section{3. fMRI results: dorsal regions of interest}

\subsubsection{Intraparietal sulcus}

A two-way ANOVA revealed a significant group $\times$ stimulus type interaction $(F(2,40)=29.59, p<0.001)$. Follow-up one-way ANOVAs revealed significant main effects of group for both the aversive and neutral scenes $(F(1,20)=$ $7.50, p<0.013$, and $F(1,20)=13.13, p<0.002$, respectively).
Group 2, for whom aversive scenes were task-relevant, generated a larger response to both the aversive and neutral scenes than Group 1. A follow-up one-way ANOVA for the circles also revealed a significant main effect $(F(1,20)=$ $-46.27, p<0.001$ ) but in the opposite direction-Group 1, for whom circles were task-relevant, generated a larger response than Group 2 (see Fig. 3).

\subsubsection{Middle frontal gyrus}

A two-way ANOVA revealed a significant group $\times$ stistimulus type interaction $(F(2,40)=9.26, p<0.001)$. Follow-up one-way ANOVAs revealed a marginally significant main effect of group for both the aversive and neutral scenes $(F(1,20)=4.25, p<0.052$, and $F(1,20)=6.73, p<0.017$, respectively), with Group 2 generating a larger response than 


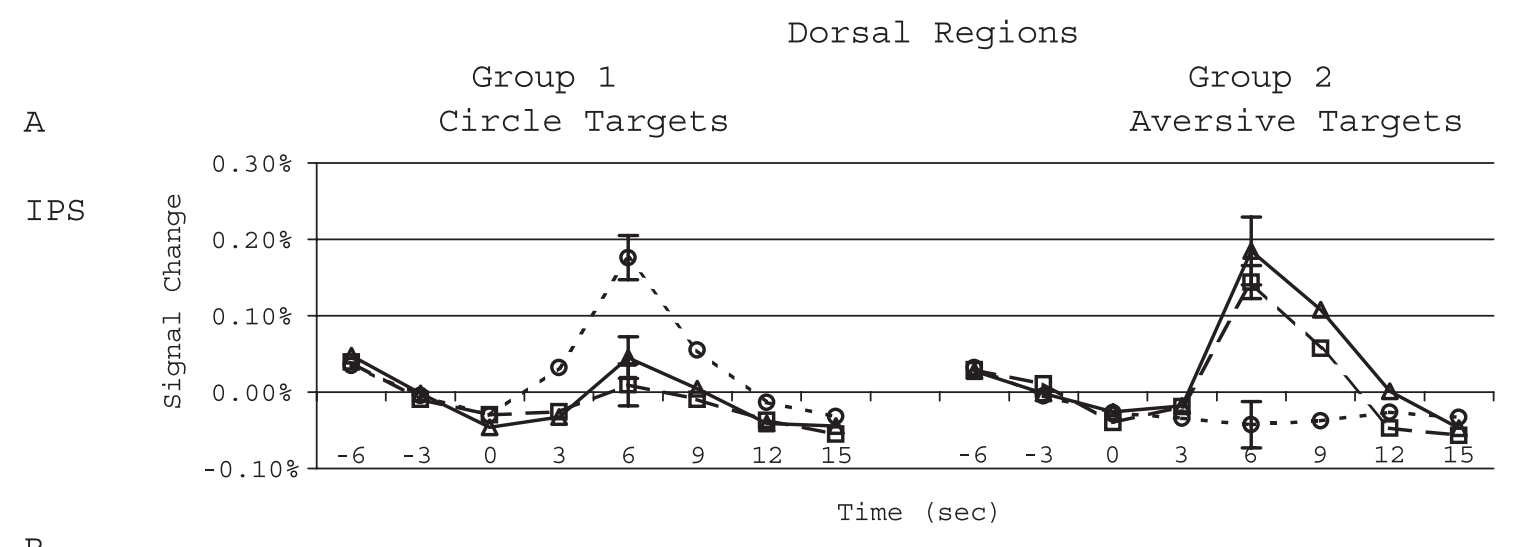

B

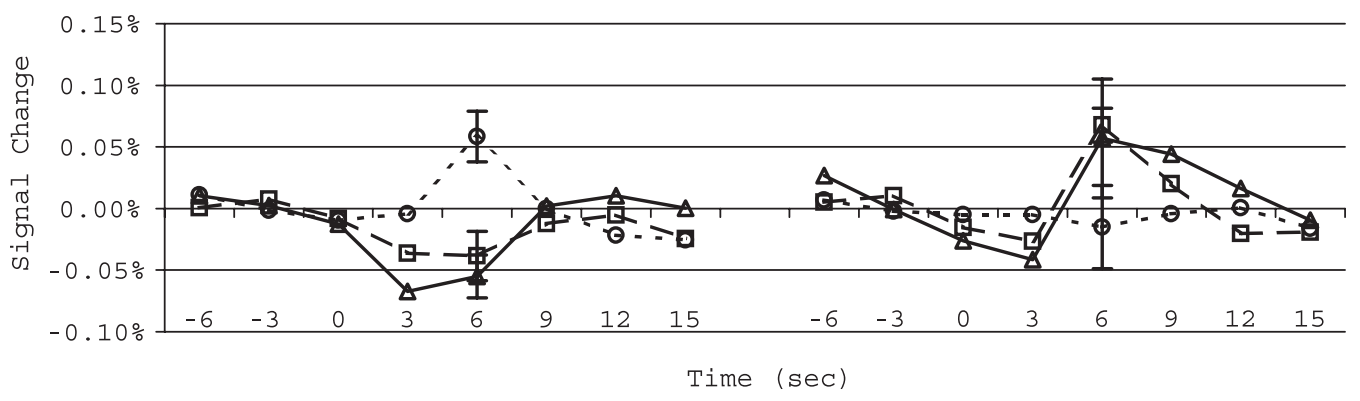

C

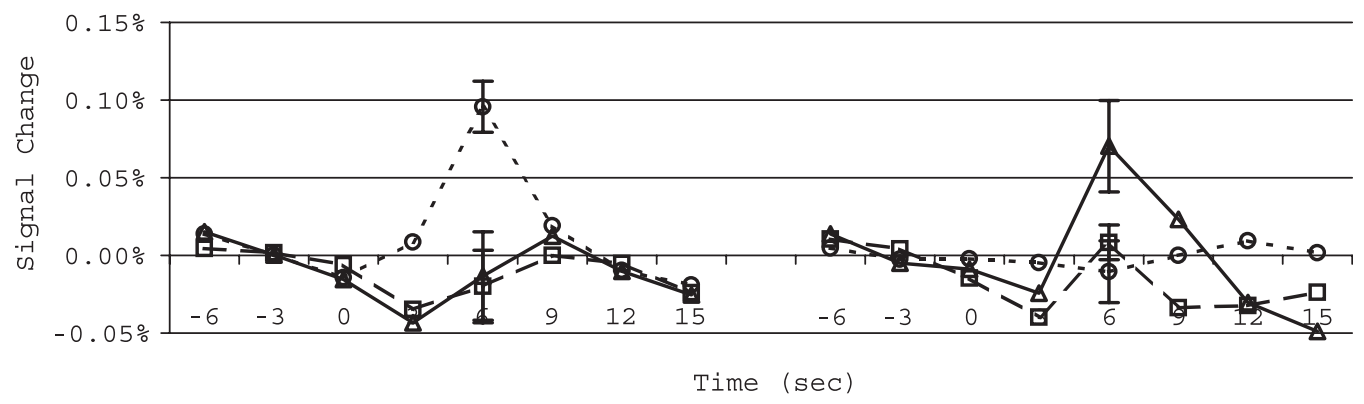

$\triangle$ Aversive Scenes $\quad$ - $₫$ - Neutral scenes $\quad$ - - - - Circles

Fig. 3. Activation in the dorsal regions of interest by group averaged across left and right hemispheres. Circles were task-relevant for Group 1, whereas aversive scenes were task-relevant for Group 2. Response within the intraparietal sulcus (A), middle frontal gyrus (B), and supramarginal gyrus (C) to circles, aversive scenes, and neutral scenes is depicted.

Group 1. A follow-up one-way ANOVA for the circles also revealed a significant main effect of group $(F(1,20)=4.94$, $p<0.038$ ), but in the opposite direction-Group 1 generated a larger response than Group 2 (see Fig. 3).

\subsubsection{Supramarginal gyrus}

A two-way ANOVA revealed a significant group $\times$ stimulus type interaction $(F(2,40)=14.26, p<0.001)$. A follow-up one-way ANOVA for the aversive scenes revealed a significant main effect of group $(F(1,20)=5.08, p<0.036)$, with Group 2 generating a larger response than Group 1. A follow-up one-way ANOVA for the circles revealed a significant main effect of group $(F(1,20)=41.21, p<$ 0.001 ), with Group 1 generating a larger response than Group 2. A follow-up one-way ANOVA for the neutral scenes revealed no significant effects $(F(1,20)=0.97, p=$ 0.336; see Fig. 3).

\section{4. fMRI results: cingulate gyrus}

The CG was split into four regions along its rostrocaudal extent. The most anterior region (1) extended from 1.875 to $0.75 \mathrm{~cm}$ anterior to the AC. The second most anterior region (2) extended from $0.375 \mathrm{~cm}$ anterior to the $\mathrm{AC}$ to $0.75 \mathrm{~cm}$ posterior to the AC. The second most posterior region (3) extended from 1.125 to $2.25 \mathrm{~cm}$ posterior to the AC. The most posterior region (4) extended from 2.625 to $3.75 \mathrm{~cm}$ posterior to the AC. Regions (1) and (2) encompassed Brodmann's area 24, whereas regions (3) and (4) encompassed Broadmann's area 23 ([51]; see Fig. 4 inset). 
A

Cingulate Gyrus

Anterior Cingulate

Posterior Cingulate

Group 1
(Circle
Target)

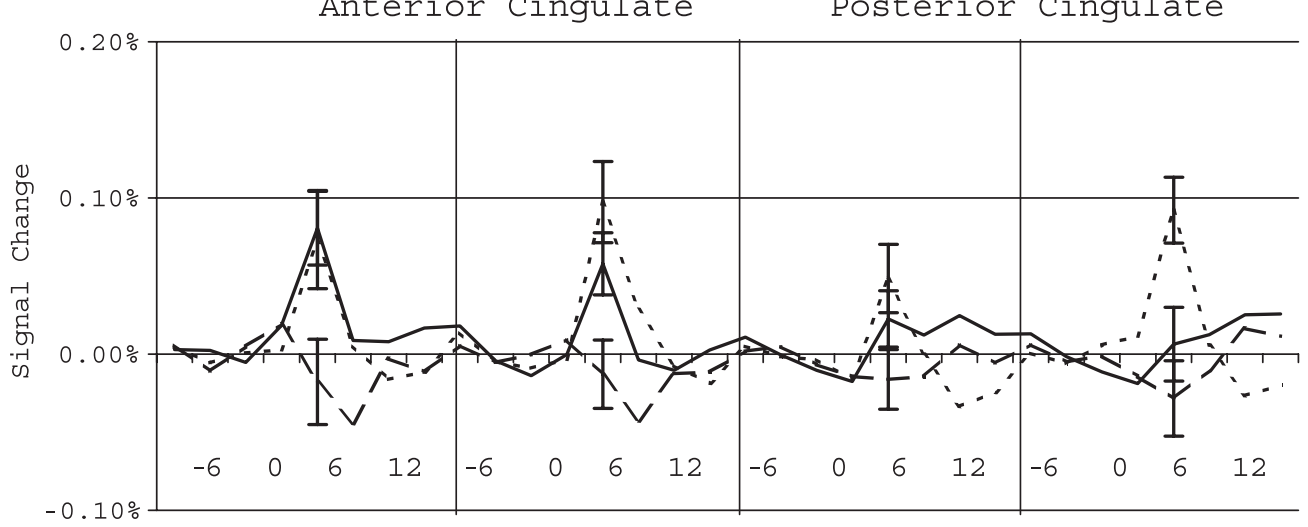

B

Region (1)

(2)

(3)

(4)

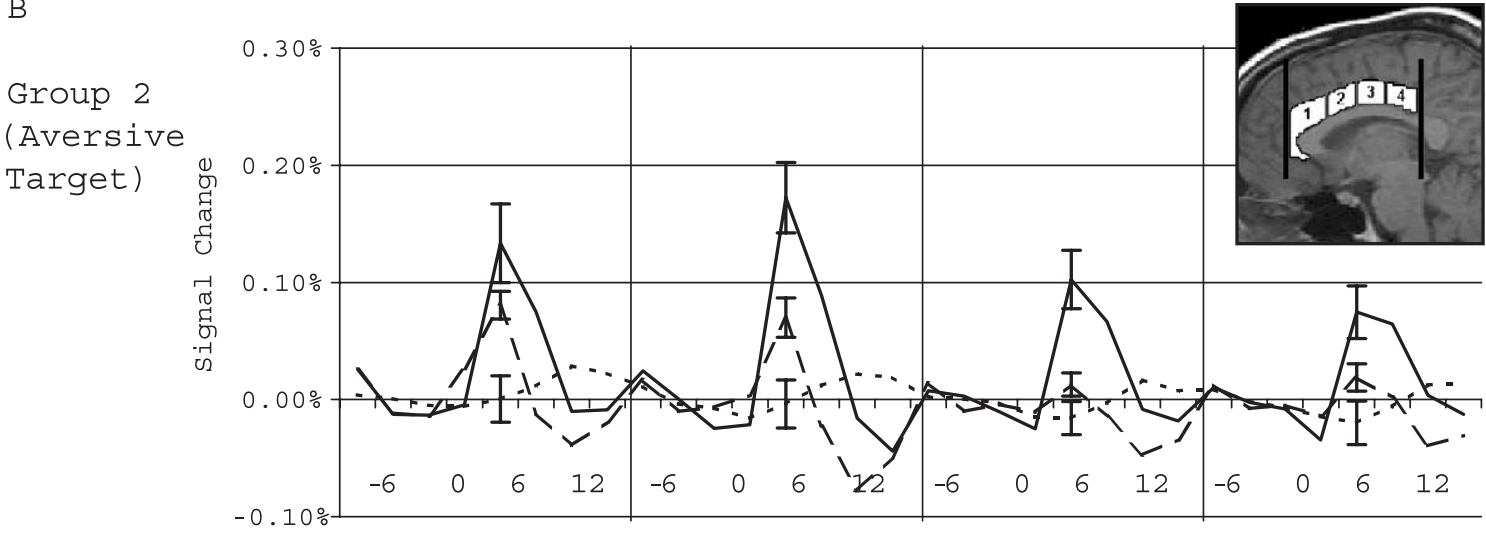

Aversive Scenes - - Neutral Scenes -..-. Circles

C

Peak Activation in Anterior and Posterior Cingulate Gyrus

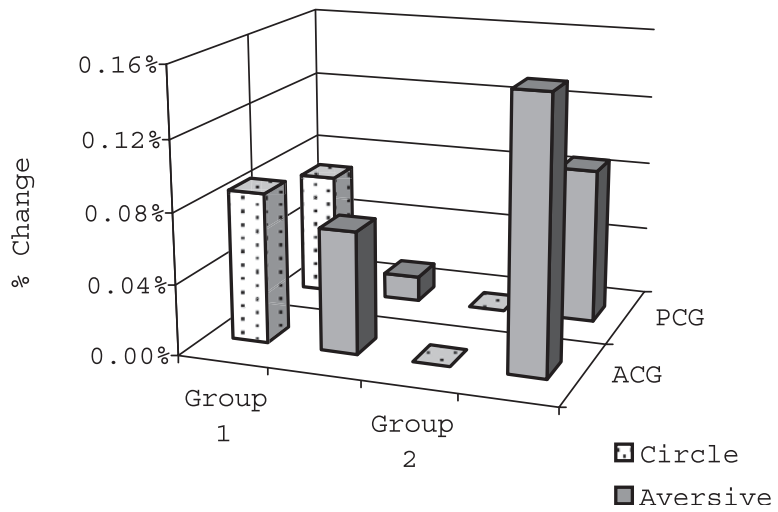

Fig. 4. Distribution of cingulate cortex activation along its rostrocaudal extent, numbered in sequential sectors from most anterior (1) to most posterior (4). Activation to circles, aversive scenes, and neutral scenes is depicted for (A) Group 1 (circle targets) and (B) Group 2 (aversive scene targets). (C) Peak activation to circles and aversive scenes within the anterior and posterior cingulate by group. Anteriorly, the response in Group 1 to task-irrelevant aversive scenes is equivalent to that for task-relevant circles, and the activation to task-relevant aversive scenes (Group 2) is greater that that seen to either stimulus category in Group 1. Posteriorly, there is only a response to the task-relevant attentional targets, which is equivalent across the two groups. (Inset) Depiction of the location of the four ROIs within the cingulate.

Paracingulate regions BAs 31 and 32 were not included in the ROI analysis but are, of course, included in the wholebrain SPM99 analysis.
The CG was characterized by a number of distinct patterns of activation. First, as expected, the response to aversive scenes was greater in Group 2 than in Group 1. 
Second, the responses in the anterior regions $(1,2)$ were greater than the responses in the posterior regions $(3,4)$ for both groups.

Group 1 showed three patterns of responses in the CG. The anterior regions $(1,2)$ showed equal activation for the task-relevant circles and aversive scene distracters, which were both greater than the activation to neutral scene distracters. The second most posterior region (3) showed greater activation to task-relevant circles than neutral scenes, while neither differed from the aversive scenes. The most posterior region (4) showed greater activation to the task-relevant circles than aversive or neutral scenes, which did not differ from each other.

Group 2 showed two patterns of activation in the CG. In the anterior regions $(1,2)$, the response to task-relevant aversive scenes was greater than that to neutral scenes, which, in turn, was greater than that to circles. In the posterior regions $(3,4)$, the response to task-relevant aversive scenes was greater than that to neutral scenes and circles, which did not differ from each other. These results are shown in Fig. 4, and the statistical details are described below.

A three-way ANOVA revealed a significant group $\times$ stimulus type $\times$ region interaction $(F(6,120)=3.38, p<$ $0.004)$. Follow up analyses revealed a significant stimulus type $\times$ region interaction for both groups $(F(6,54)=4.45$, $p<0.001$ for Group 1 and $F(6,66)=3.21, p<0.008$ for Group $2)$. The effect of stimulus type was investigated within each region by group.

For Group 1, where circles were task-relevant, there was a significant effect of stimulus type in each region $(F(2,27)=4.48, p<0.021$ for region $1, F(2,27)=7.73$, $p<0.003$ for region $2, F(2,27)=3.64, p<0.039$ for region 3 , and $F(2,27)=8.91, p<0.001$ for region 4$)$. In the most anterior region (1), the activation to aversive scenes and circles did not differ, and both were significantly greater than the activation to neutral scenes. This pattern was also seen in the second most anterior region (2). In the second most posterior region (3), the activation to aversive scenes did not differ from the activation to circles or neutral scenes, but the activation to the circles was significantly greater that the activation to the neutral scenes. In the most posterior region (4), the activation to circles was significantly greater than the activation to aversive and neutral scenes, which did not differ.

For Group 2, where aversive scenes were task-relevant, there was a significant effect of stimulus type in each region $(F(2,33)=18.68, p<0.001$ for region $1, F(2,33)=$ $16.47, p<0.001$ for region $2, F(2,33)=16.16, p<0.001$ for region 3 , and $F(2,33)=8.70, p=0.001$ for region 4$)$. In the most anterior region (1), the activation to aversive scenes was significantly greater that to neutral scenes, which, in turn, was significantly greater than the activation to circles. This pattern was also seen in the second most anterior region (2). In the second most posterior region (3), the activation to aversive scenes was significantly greater than the activation to circles and neutral scenes, which did not differ. This pattern was also seen in the most posterior region (4).

An additional planned comparison investigated the effects of region and attention on the response to aversive scenes. This group $\times$ region ANOVA revealed significant main effects of group $(F(1,20)=11.47, p<0.003)$ and region $(F(3,60)=9.26, p<0.001)$. Across all regions, Group 2, where the aversive scenes were task-relevant, had greater activation to the aversive scenes than Group 1. Post hoc contrasts showed that the two anterior regions $(1,2)$ had a greater response to the aversive scenes than the posterior regions $(3,4)$ across groups.

\section{5. fMRI results: hemispheric asymmetries}

Although not the primary aim of the study, we conducted additional analyses to investigate the effects of hemisphere, given the importance of cerebral dominance in theories of emotion and attention $[5,38,47]$. Three regions showed hemispheric effects, but none of these involved responses to the aversive or neutral distracters. Within the IFG, there were significant group $\times$ hemisphere $(F(1,20)=6.12, p<$ $0.022)$ and stimulus type $\times$ hemisphere $(F(2,40)=3.31$, $p<0.047)$ interactions. Post hoc contrasts showed that the right hemisphere in Group 1 had a larger response than the left to the task-relevant circles. Activation in the IPS showed a larger response in the right hemisphere regardless of stimulus type or group $(F(1,20)=6.08, p<0.023)$. Within the MFG, there was a significant stimulus type $\times$ hemisphere interaction $(F(2,40)=3.88, p<0.029)$. Post hoc

Table 2

Results of conjunction analyses showing common regions of activation for aversive scenes and attentional targets across groups

\begin{tabular}{|c|c|c|c|c|}
\hline Region of activation & Side & $X$ & $Y$ & $Z$ \\
\hline \multicolumn{5}{|l|}{ Aversive scenes } \\
\hline \multirow[t]{2}{*}{ Amygdala } & $\mathrm{L}$ & -21.0 & -3.0 & -18.0 \\
\hline & $\mathrm{R}$ & 23.0 & -3.0 & -18.0 \\
\hline \multirow[t]{2}{*}{ Fusiform gyrus } & $\mathrm{L}$ & -41.0 & -67.0 & -13.0 \\
\hline & $\mathrm{R}$ & 41.0 & -67.0 & -13.0 \\
\hline \multirow[t]{2}{*}{ Inferior frontal gyrus } & $\mathrm{L}$ & -47.0 & 18.0 & -8.0 \\
\hline & $\mathrm{R}$ & 50.0 & 20.0 & -9.0 \\
\hline Medial prefrontal cortex & $\mathrm{L}$ & -1.0 & 60.0 & 18.0 \\
\hline \multirow[t]{2}{*}{ Parahippocampal gyrus } & $\mathrm{L}$ & -20.0 & -25.0 & -13.0 \\
\hline & $\mathrm{R}$ & 22.0 & -23.0 & -13.0 \\
\hline Superior frontal gyrus & $\mathrm{L}$ & -1.0 & 21.0 & 50.0 \\
\hline \multicolumn{5}{|l|}{ Attentional targets } \\
\hline Cingulate gyrus & $\mathrm{R}$ & 2.0 & 17.0 & 39.0 \\
\hline \multirow[t]{2}{*}{ Postcentral gyrus } & $\mathrm{L}$ & -48.0 & -32.0 & 52.0 \\
\hline & $\mathrm{R}$ & 50.0 & -32.0 & 52.0 \\
\hline Superior frontal gyrus & $\mathrm{R}$ & 1.0 & 10.0 & 50.0 \\
\hline \multirow[t]{2}{*}{ Superior temporal gyrus } & $\mathrm{L}$ & -52.0 & 10.0 & -5.0 \\
\hline & $\mathrm{R}$ & 52.0 & 13.0 & -5.0 \\
\hline \multirow[t]{2}{*}{ Thalamus } & $\mathrm{L}$ & -10.0 & -6.0 & 10.0 \\
\hline & $\mathrm{R}$ & 13.0 & -5.0 & 10.0 \\
\hline
\end{tabular}

All activated clusters are significant at $p<0.001$ with a minimum spatial extent of four voxels. Coordinates of activated clusters are reported in relation to the stereotaxic system described by Talairach and Tournoux [52]. 


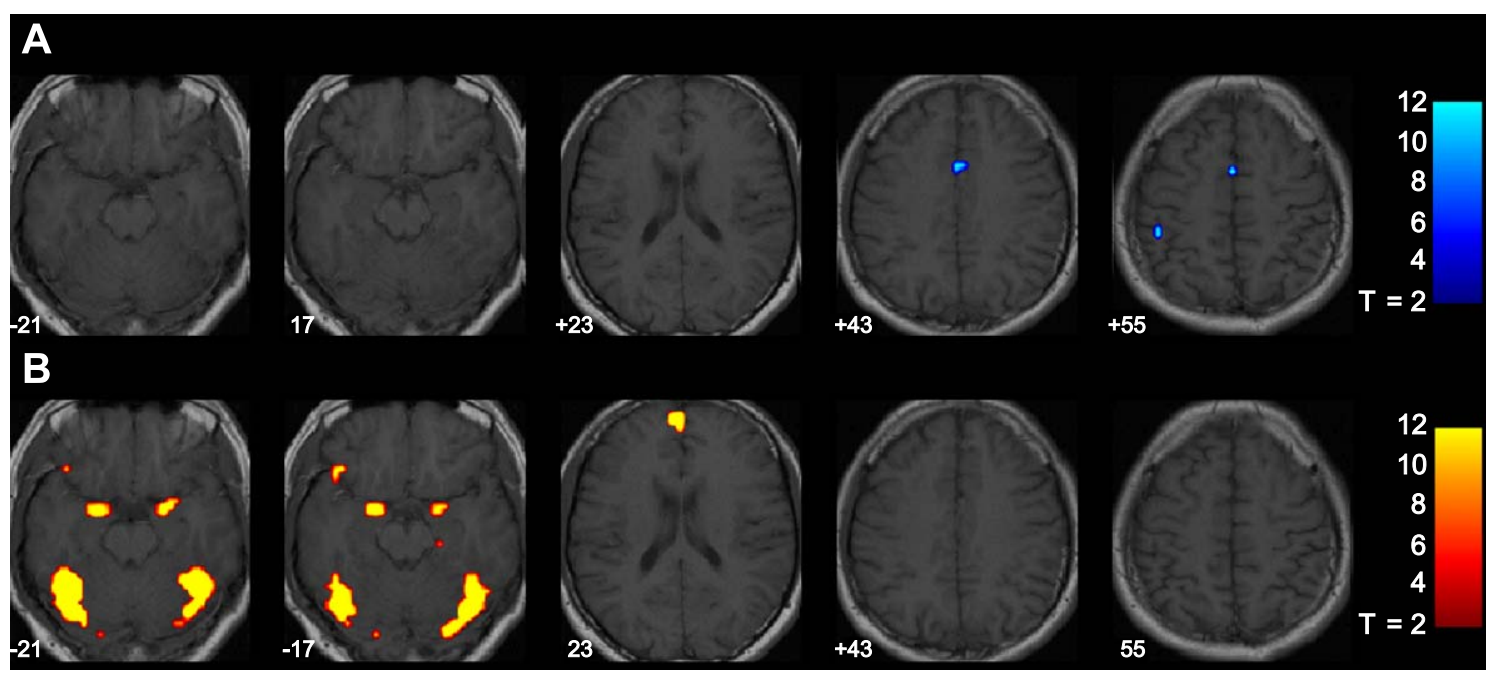

Fig. 5. Statistical parametric maps from the voxelwise conjunction analyses across groups. (A) Conjunction of attentional targets across groups (circles from Group 1 and aversive scenes from Group 2), $p<0.001$ uncorrected within each group. (B) Conjunction analysis of aversive scenes across groups, $p<0.001$ uncorrected within each group. Talairach coordinates of all activated regions are provided in Table 2.

contrasts showed that the right hemisphere showed larger activation than the left to the circles.

To summarize, in each of these regions the response in the right hemisphere was greater than the left hemisphere. In the IPS this was true for all stimulus types across groups, but in the IFG and MFG the hemispheric asymmetry was only apparent in response to the task-relevant circles (Group 1). In the IFG, the response to the circles by Group 1 went below baseline in the post-stimulus period, which accounts for the hemispheric asymmetry effect.

\section{6. fMRI results: SPM analysis}

Two conjunction analyses were performed to determine the regions that were responsive to (a) the emotional content of a stimulus (regardless of task relevancy) and (b) the taskrelevance of a stimulus (regardless of emotional content). The results of these analyses are presented in Table 2 and Fig. 5.

The conjunction of aversive scenes across both groups revealed activation bilaterally in the amygdala, FFG, IFG, and parahippocampal gyrus. There was also activation in the left medial prefrontal cortex and left superior frontal gyrus. The conjunction of attentional targets across both groups revealed activation bilaterally in the postcentral gyrus, superior temporal gyrus and thalamus, as well as activation in the right ACG and superior frontal gyrus.

\section{Discussion}

Using a visual oddball task, the current study demonstrated that a network of regions in the ventral portion of the brain were sensitive to aversive emotional content irrespective of attentional focus, as defined by task rele- vancy. These brain areas included the AMG, IFG, FFG, parahippocampal gyrus, and visual association cortices. An additional dorsal network of brain regions was sensitive to the attentional focus placed upon task-relevant stimuli irrespective of their emotional content. These brain areas included the IPS, SMG, MFG, and PCG. The ACG contributed to both attentional and emotional functions. The response in the ACG was equivalent to both taskrelevant targets and emotionally salient distracters in Group 1 , where attentional and emotional processing were distributed across different stimulus categories over time. In Group 2, where attentional and emotional processing converged on a common stimulus target, the response in the ACG was super-additive relative to that observed for Group 1. This pattern of results supports anatomical theories suggesting that the anterior cingulate serves to integrate emotional and attentional streams of processing in the brain $[34,37,43]$. Below, we discuss the findings relative to other studies of emotional scene encoding and attentional oddball tasks.

\subsection{Ventral regions}

Emotional scene encoding is known to engage multiple stages of visual processing along the ventral stream as well as fronto-limbic structures $[29,32,44,53]$. The common areas of activation across studies include the extrastriate cortex, AMG, FFG, and IFG. The present study showed that activity along these ventral regions was similar across the two experimental groups despite longer RTs when the emotional scenes were task relevant. These brain regions thus appear to be insensitive to time-on-task when accuracy is equated. In a review of this literature, Phan et al. [46] suggest that these ventral regions may be active specifically in response to the visual presentation of emotional informa- 
tion and the induction of fear by these stimuli (specifically activation in the amygdala). They also suggest that the ventral fronto-limbic and occipital regions are active in response to the emotional content of the stimulus regardless of which stimulus has the attentional focus during the task. The current results provide direct support for this claim. Similar findings have been reported in the amygdala during attentional studies of fearful facial expressions $[3,56]$. However, other research suggests that amygdalar processing of facial expressions is enhanced when the emotion is taskrelevant $[18,19]$ or under conditions of high attentional load [45]. Still other studies have shown enhancement of amygdala responses under passive, subliminal, or unattended processing conditions ([3]— disgust expression, [40,54]). It is not yet clear which factors contribute to these discrepant results (stimulus type, emotional category, attentional demands, task design, anatomical locus, etc.).

The present study also revealed a suppression of left IFG activity to circles when they were attentional targets (Group 1) but not when aversive scenes were attentional targets (Group 2). There was also a trend towards suppression of MFG activity to the aversive scenes when the circles were attentional targets (Group 1) but not when the scenes were the attentional targets (Group 2). In both cases, the responses were below baseline during the post-stimulus period. In other words, different compartments within the prefrontal cortex that primarily index cognitive and emotional functions, respectively, appeared to interact differently according to whether attentional and emotional processing were distributed across objects over time or were focused concurrently on the same stimulus category. This pattern is consistent with the observation by Drevets and Raichle [13] that neural activity may be reduced in areas responsible for emotional processing during cognitive operations and vice versa (i.e., Group 1 showed IFG deactivations to circle targets but simultaneous MFG activations to the same stimuli). Although the orbitofrontal cortex is also an important prefrontal component for emotional processing, signal dropout due to susceptibility artifacts prevented our ability to image this area using conventional EPI imaging (see Ref. [26]).

\subsection{Dorsal regions}

The dorsal frontoparietal regions engaged by attentional targets in the present study are consistent with those reported in previous oddball tasks $[4,8,9,25,35,49]$. Furthermore, we show here that these dorsal regions respond to task-relevant oddball stimuli similarly despite variations in their emotional properties.

Previous oddball tasks that have included task-irrelevant distracters in the experimental design have reported mixed results with respect to neural processing of stimulus probability $[8,9,25]$. In contrast to the current study, where no dorsal activation was seen in response to the distracters, Clark et al. [9] showed activity in left superior parietal gyrus and right postcentral gyrus to distracting stimuli. Other studies have described results more consistent with the current findings, with no significant activation in dorsal regions to distracting stimuli but a trend towards significance in the medial prefrontal cortex and anterior cingulate $[8,25]$. Despite some variability in distracter-related activation, there is much consistency across studies with respect to dorsal stream processing of attentional (oddball) targets and their characteristic electrophysiological signature, namely a P300 response (reviewed in Ref. [17]). The frontoparietal activations are also consistent with other studies in which attention was distributed across time rather than space [10].

Finally, we comment on the lack of dorsal stream processing to emotional stimuli when they are not taskrelevant. Although some studies have shown parietal cortex activation during emotional processing tasks [29,41,48, $50,57,59]$, the present study raises the question of whether attentional demands are driving such effects. Some of the studies showing dorsal activation have used tasks in which the emotional information is task-relevant, which may have consequently introduced a confound between emotional and attentional factors. Others have compared passive viewing to an active task condition where the two conditions are not equally demanding. Lane et al. [28] also showed greater parietal lobe responses to aversive scenes when participants evaluated their spatial setting rather than their emotional content. This finding supports the results of the present study regarding the influence of task relevancy on recruiting dorsal stream responses to emotional stimuli.

\subsection{Emotion and attention: cingulate gyrus}

Previous researchers have divided the ACG into functionally distinct regions, with more rostral and ventral areas subserving emotional functions and more caudal and dorsal areas subserving cognitive functions $[12,55]$. These distinctions have been supported by recent functional imaging studies largely using Stroop interference paradigms where emotion and attention conflict in a task-relevant manner $[6,59]$. The present study took a different approach to the topic of attentional and emotional representation within the cingulate gyrus. Namely, we examined how cingulate activity varied when emotional and attentional processing were distributed in time (Group 1) and conjointly engaged (Group 2 ). By comparing activity across the experimental groups, one can understand how the cingulate signals these functions both in isolation and in combination.

The ROI analysis divided the cingulate gyrus into four regions along its rostrocaudal extent. The results implicate a functional boundary between the first two and last two regions. The anterior regions $(1,2)$ encompassing BA 24 responded equally to the attentional targets (circles) and the emotional distracters for Group 1. Importantly, these regions more than doubled their activity in response to emotional stimuli in Group 2, for whom the emotional 
scenes served as attentional targets. In contrast, the posterior regions $(3,4)$ encompassing BA 23 were only engaged to the attentional targets regardless of emotional content. The results show clear differences between anterior and posterior divisions of the cingulate, but they do not support a dissociation between affective and cognitive subdivisions within the ACG [7]. Our conclusions are limited, though, by the resolution of the methods employed. The ROIs were drawn in coronal section and may not have been ideal for extracting such subdivisions of the ACG. Nonetheless, the analysis showed that even rostral BA 24, which is hypothesized to lie in the affective division of ACG, was sensitive to both emotional and attentional factors. The voxelwise SPM analysis did suggest a dorsal-ventral gradient in the location of the most activated (peak) voxels, with circle targets engaging more dorsal areas of the ACG. However, peak activity to emotional scenes was in front of ACG proper, located in Brodmann area $9 / 10$, and there was additional activity to the aversive scenes in a dorsal region of the ACG (Fig. 5). Thus, the affective and cognitive subdivisions of the anterior cingulate may not be separable under all task conditions. Instead, the findings support anatomic theories that the ACG is a site where the integration of the emotional value and the task-relevancy of a stimulus are combined $[2,34,37,43]$.

\subsection{Limitations}

The present study has three main limitations. First, only aversive scenes were used as emotional stimuli. Therefore, the relative contributions of arousal and valence could not be ascertained. Second, because of the small sample sizes, we could not analyze the influence of gender on brain activation patterns. Third, the attentional demands placed upon participants may have differed across the two conditions, despite similar dorsal stream responses to aversive scenes across groups. This is reflected both in the increased RTs to scenes in Group 2 (Table 1) and in the partial recruitment of dorsal stream regions to neutral scenes in Group 2 (Fig. 3). This possible difference in attentional demands is an unavoidable consequence of varying the assignment of target stimuli across the two groups while keeping all other task parameters the same. Although detection accuracy was equivalent across groups, future studies should strive to equate both accuracy and RTs across groups.

\subsection{Conclusions}

The present study provides insight into the interactions between emotional and attentional processing networks in the brain. Viewing emotional material, regardless of task relevancy, elicited activation in ventral stream regions, including the amygdala and higher-level extrastriate cortex. Responding to task-relevant stimuli, irrespective of their emotional content, elicited activation in dorsal frontoparietal and posterior cingulate areas known to be involved in attentional control. Activation in the ACG was sensitive to both attentional and emotional factors and potentially serves as a critical node linking these two functions. Not only was the ACG responsive to both aversive scenes and attentional targets when presented intermittently (Group 1), but its activity more than doubled when the aversive scenes became the attentional targets (Group 2). These findings support and extend prominent network theories of emotion-attention interactions that highlight the integrative role played by the anterior cingulate.

\section{Acknowledgements}

This work was supported by National Institutes of Health grants P50 MH60451 and R01 DA14094, a Young Investigator Award from the National Alliance for Research on Schizophrenia and Depression, and a Ralph E. Powe Junior Faculty Enhancement Award from Oak Ridge Associated Universities.

\section{References}

[1] R. Adolphs, Neural systems for recognizing emotion, Curr. Opin. Neurobiol. 12 (2002) 169-177.

[2] J.M. Allman, A. Hakeem, J.M. Erwin, E. Nimchinsky, P. Hof, The anterior cingulate cortex: the evolution of an interface between emotion and cognition, Ann. N. Y. Acad. Sci. 935 (2001) 107-117.

[3] A.K. Anderson, K. Christoff, D. Panitz, E. De Rosa, J.D.E. Gabrieli, Neural correlates of the automatic processing of threat facial signals, J. Neurosci. 23 (2003) 5627-5633.

[4] B.A. Ardekani, S.J. Choi, G.-A. Hossein-Zadeh, B. Porjesz, J.L. Tanabe, K.O. Lim, R. Bilder, J.A. Helpern, H. Begleiter, Functional magnetic resonance imaging of brain activity in the visual oddball task, Cogn. Brain Res. 14 (2002) 347-356.

[5] J.C. Borod, B.A. Cicero, L.K. Obler, J. Welkowitz, H.M. Erhan, C. Santschi, I.S. Grunwald, R.M. Agosti, J.R. Whalen, Right hemisphere emotional perception: evidence across multiple channels, Neuropsychology 12 (1998) 446-458.

[6] G. Bush, P.J. Whalen, B.R. Rosen, M.A. Jenike, S.C. McInerney, S.L. Rauch, The counting Stroop: an interference task specialized for functional neuroimaging-validation study with functional MRI, Hum. Brain Mapp. 6 (1998) 270-282.

[7] G. Bush, P. Luu, M.I. Posner, Cognitive and emotional influences in anterior cingulate cortex, Trends Cogn. Sci. 4 (2000) 215-222.

[8] V.P. Clark, S. Fannon, S. Lai, R. Benson, L. Bauer, Responses to rare visual target and distractor stimuli using event-related fMRI, J. Neurophysiol. 83 (2000) 3133-3139.

[9] V.P. Clark, S. Fannon, S. Lai, R. Benson, Paradigm-dependant modulation of event-related fMRI activity evoked by the oddball task, Hum. Brain Mapp. 14 (2001) 116-127.

[10] J.T. Coull, A.C. Nobre, Where and when to pay attention: the neural systems for directing attention to spatial locations and to time intervals as revealed by both PET and fMRI, J. Neurosci. 18 (1998) $7426-7435$.

[11] E. Courchesne, S.A. Hillyard, R. Galambos, Stimulus novelty, task relevance and the visual evoked potential in man, Electroencephalogr. Clin. Neurophysiol. 39 (1975) 131-143.

[12] O. Devinsky, M.J. Morrell, B.A. Vogt, Contributions of anterior cingulate cortex to behaviour, Brain 118 (1995) 279-306. 
[13] W.C. Drevets, M.E. Raichle, Suppression of regional cerebral blood flow during emotional versus higher cognitive processes: implications for interactions between emotion and cognition, Cogn. Emot. 12 (1998) 353-385.

[14] J. Driver, R.S.J. Frackowaik, Neurobiological measures of human selective attention, Neuropsychologia 39 (2001) 1257-1262.

[15] H.M. Duvernoy, The Human Brain: Surface, Blood Supply, and Three Dimensional Sectional Anatomy, Springer-Verlag, Wien, 1999.

[16] R.S.J. Frackowiak, K.J. Friston, C.D. Frith, R.J. Dolan, J.C. Mazziotta, Human Brain Function, Academic Press, New York, 1997.

[17] D. Friedman, Y.M. Cycowicz, H. Gaeta, The novelty P3: an eventrelated brain potential (ERP) sign of the brain's evaluation of novelty, Neurosci. Biobehav. Rev. 25 (2001) 355-373.

[18] R.C. Gur, L. Schroeder, T. Turner, C. McGrath, R.M. Chan, B.I. Turetsky, D. Alsop, J. Maldjian, R.E. Gur, Brain activation during facial emotion processing, NeuroImage 16 (2002) 651-662.

[19] A.R. Hariri, S.Y. Bookheimer, J.C. Mazziotta, Modulating emotional responses: effects of a neocortical network on the limbic system, NeuroReport 11 (2000) 43-48.

[20] J.V. Haxby, C.L. Grady, B. Horwitz, L.G. Ungerleider, M. Mishkin, R.E. Carson, P. Herscovitch, M.B. Schapiro, S.I. Rapoport, Dissociation of object and spatial visual processing in human extrastriate cortex, Proc. Natl. Acad. Sci. U. S. A. 88 (1991) 1621-1625.

[21] K.M. Heilman, R.T. Watson, E. Valenstein, A.R. Damasio, Localization of lesions in neglect, in: A. Kertesz (Ed.), Localization in Neuropsychology, Academic Press, New York, 1983, pp. 455-470.

[22] S.A. Huettel, G. McCarthy, Evidence for a refractory period in the hemodynamic response to visual stimuli as measured by MRI, NeuroImage 11 (2000) 547-553.

[23] S.A. Huettel, G. McCarthy, Regional differences in the refractory period of the hemodynamic response: an event-related fMRI study, NeuroImage 14 (2001) 967-976.

[24] A.P. Jha, G. McCarthy, The influence of memory load upon delayinterval activity in a working-memory task: an event-related functional MRI study, J. Cogn. Neurosci. 12 (S2) (2000) 90-105.

[25] E. Kirino, A. Belger, P. Goldman-Rakic, G. McCarthy, Prefrontal activation evoked by infrequent target and novel stimuli in a visual target detection task: an event-related functional magnetic resonance imaging study, J. Neurosci. 20 (2000) 6612-6618.

[26] K.S. LaBar, D.R. Gitelman, M.-M. Mesulam, T.B. Parrish, Impact of signal-to-noise on functional MRI of the human amygdala, NeuroReport 12 (2001) 3461-3464.

[27] J.L. Lancaster, M.G. Woldorff, L.M. Parsons, M. Liotti, C.S. Freitas, L. Rainey, P.V. Kochunov, D. Nickerson, S.A. Mikiten, P.T. Fox, Automated Talairach Atlas labels for functional brain mapping, Hum. Brain Mapp. 10 (2000) 120-131.

[28] R.D. Lane, G.R. Fink, P.M.-L. Chau, R.J. Dolan, Neural activation during selective attention to subjective emotional responses, NeuroReport 8 (1997) 3969-3972.

[29] R.D. Lane, E.M. Reiman, M.M. Bradley, P.J. Lang, G.L. Ahern, R.J. Davidson, G.E. Schwartz, Neuroanatomical correlates of pleasant and unpleasant emotion, Neuropsychologia 35 (1997) 1437-1444.

[30] R.D. Lane, E.M. Reiman, B. Axelrod, L.-S. Yun, A. Holmes, G.E. Schwartz, Neural correlates of levels of emotional awareness: evidence of an interaction between emotion and attention in the anterior cingulate cortex, J. Cogn. Neurosci. 10 (1998) 525-535.

[31] R.D. Lane, P.M.-L. Chua, R.J. Dolan, Common effects of emotional valence, arousal and attention on neural activation during visual processing of pictures, Neuropsychologia 37 (1999) 989-997.

[32] P.J. Lang, M.M. Bradley, J.R. Fitzsimmons, B.N. Cuthbert, J.D. Scott, B. Moulder, V. Nangia, Emotional arousal and activation of the visual cortex: an fMRI analysis, Psychophysiology 35 (1998) 199-210.

[33] P.J. Lang, M.M. Bradley, B.N. Cuthbert, International Affective Pictures System (IAPS): Instruction Manual and Affective Ratings, The Center for Research in Psychophysiology, University of Florida, Gainesville, FL, 2001.
[34] H.S. Mayberg, Limbic-cortical dysregulation: a proposed model of depression, J. Neuropsychiatry Clin. Neurosci. 9 (1997) 471-481.

[35] G. McCarthy, M. Luby, J. Gore, P. Goldman-Rakic, Infrequent events transiently activate human prefrontal and parietal cortex as measured by functional MRI, J. Neurophysiol. 77 (1997) 1630-1634.

[36] M.S. Mega, J.L. Cummings, S. Salloway, P. Malloy, The limbic system: an anatomic, phylogenetic, and clinical perspective, J. Neuropsychiatry Clin. Neurosci. 9 (1997) 315-330.

[37] M.-M. Mesulam, A cortical network for directed attention and unilateral neglect, Ann. Neurol. 10 (1981) 309-325.

[38] M.-M. Mesulam, Patterns in behavioral neuroanatomy: association areas, the limbic system, and hemispheric specialization, in: M.-M. Mesulam (Ed.), Principles of Behavioral Neurology, Davis, Philadelphia, 1985, pp. 1-70.

[39] M.-M. Mesulam, Spatial attention and neglect: parietal, frontal and cingulate contributions to the mental representation and attentional targeting of salient extrapersonal events, Philos. Trans. R. Soc., B 354 (1999) $1325-1346$.

[40] J.S. Morris, A. Ohman, R.J. Dolan, Conscious and unconscious emotional learning in the human amygdala, Nature 393 (1998) 467-470.

[41] J. Narumoto, T. Okada, N. Sadato, K. Fukui, Y. Yonekura, Attention to emotion modulates fMRI activity in human right superior temporal sulcus, Cogn. Brain Res. 12 (2001) 225-231.

[42] E. Nimchinsky, E. Gilissen, J.M. Allman, D.P. Perl, J.M. Erwin, P.R. Hof, A neuronal morphologic type unique to humans and great apes, Proc. Natl. Acad. Sci. U. S. A. 96 (1999) 5268-5273.

[43] J.W. Papez, A proposed mechanism of emotion, Arch. Neurol. Psychiatry 38 (1937) $725-742$.

[44] S. Paradiso, D.L. Johnson, N.C. Andreasen, D.S. O'Leary, G.L. Watkins, L.L. Boles Ponto, R.D. Hichwa, Cerebral blood flow changes with attribution of emotional valence to pleasant, unpleasant, and neutral visual stimuli in a PET study of normal subjects, Am. J. Psychiatry 156 (1999) $1618-1629$.

[45] L. Pessoa, M. McKenna, E. Gutierrez, L.G. Ungerleider, Neural processing of emotional faces requires attention, Proc. Natl. Acad. Sci. U. S. A. 99 (2002) 11458-11463.

[46] K.L. Phan, T. Wager, S.F. Taylor, I. Liberzon, Functional neuroanatomy of emotion: a meta-analysis of emotion activation studies in PET and fMRI, NeuroImage 16 (2002) 331-348.

[47] G.E. Schwartz, R.J. Davidson, F. Maer, Right hemisphere lateralization for emotion in the human brain: interactions with cognition, Science 190 (1975) 286-288.

[48] J.R. Simpson, D. Ongur, E. Akbudak, T.E. Conturo, J.M. Ollinger, A.Z. Snyder, D.A. Gusnard, M.E. Raichle, The emotional modulation of cognitive processing: an fMRI study, J. Cogn. Neurosci. 12 (2000) $157-170$

[49] A.A. Stevens, P. Skudlarski, J.C. Gatenby, J.C. Gore, Event-related fMRI of auditory and visual oddball tasks, Magn. Reson. Imaging 18 (2000) 495-502.

[50] B.A. Strange, R.N.A. Henson, K.J. Friston, R.J. Dolan, Brian mechanisms for detecting perceptual, semantic, and emotional deviance, NeuroImage 12 (2000) 425-433.

[51] T. Takahashi, Y. Kawasaki, K. Kurokawa, H. Hagino, S. Nohara, I. Yamashita, K. Nakamura, M. Murata, M. Matsui, M. Suzuki, H. Seto, M. Kurachi, Lack of normal structural asymmetry of the anterior cingulate gyrus in female patients with schizophrenia: a volumetric magnetic resonance imaging study, Schizophr. Res. 55 (2002) 69-81.

[52] J. Talairach, P. Tournoux, Co-Planar Stereotaxic Atlas of the Human Brain, Thieme Medical Publishers, New York, 1988.

[53] S.F. Taylor, I. Liberzon, L.M. Fig, L.R. Decker, S. Minoshima, R.A. Koeppe, The effect of emotional content on visual recognition memory: a PET activation study, NeuroImage 8 (1998) 188-197.

[54] S.F. Taylor, K.L. Phan, L.R. Decker, I. Liberzon, Subjective rating of emotionally salient stimuli modulates neural activity, NeuroImage 18 (2003) 650-659.

[55] B.A. Vogt, E.A. Nimchinsky, L.J. Vogt, P.R. Hof, Human cingulate 
cortex: surface features, flat maps, and cytoarchitecture, J. Comp. Neurol. 359 (1995) 490-506.

[56] P. Vuilleumier, J.L. Armony, J. Driver, R.J. Dolan, Effects of attention and emotion on face processing in the human brain: an event-related fMRI study, Neuron 30 (2001) 829-841.

[57] P. Vuilleumier, J.L. Armony, K. Clarke, M. Husain, J. Driver, R.J. Dolan, Neural response to emotional faces with and without awareness: event-related fMRI in a parietal patient with visual extinction and spatial neglect, Neuropsychologia 40 (2002) 2156-2166.
[58] R.T. Watson, K.M. Heilman, J.C. Cauthen, F.A. King, Neglect after cingulectomy, Neurology 23 (1973) 1003-1007.

[59] P.J. Whalen, G. Bush, R.J. McNally, S. Wilhelm, S.C. McInerney, M.A. Jenike, S.L. Rauch, The emotional counting Stroop paradigm: a functional magnetic resonance imaging probe of the anterior cingulate affective division, Biol. Psychiatry 44 (1998) 1219-1228.

[60] H. Yamasaki, K.S. LaBar, G. McCarthy, Dissociable prefrontal brain systems for attention and emotion, Proc. Natl. Acad. Sci. U. S. A. 99 (2002) 11447-11451. 